\title{
The role of regular dental examinations in the prevention of dental diseases
}

\author{
O. Ripetska, V. Hrynovets, A. Buchkovska, *V. Dovhanyk, V. Synytsia
}

Danylo Halytskyj National Medical University in Lviv, Ukraine

*Corresponding author. E-mail: viktdov@gmail.com

Paper received 17.01.20; Accepted for publication 03.02.20.

\author{
https://doi.org/10.31174/SEND-NT2020-224VIII27-11
}

\begin{abstract}
Ethic and Prophylactic Aspects of Regular Dental Examinations and Treatment in Young Patients. The number of gingivitis and periodontitis in young patients highly increased in recent decades. That is why, revelation and early treatment of periodontal diseases is important not only clinical but also ethical and psychological problem. Patient should feel himself not like a passive object in the treatment but take an active part in the process.. Being acquainted with the treatment plan, presumable duration of the treatment and its' results, helps the patient to feel more comfortable and positive about the treatment procedures.
\end{abstract}

Kaywords: dental diseases, gingivitis, periodontitis, young patients.

Introduction. Annual prophylactic dental examinations in young patients are important task in the general medical health control system. They help to reveal not only diseases of teeth but also periodontal diseases. It is also an important ethical problem to persuade young persons how important is to control the condition of oral health. In the Department of Therapeutic Dentistry of National Medical University in Lviv we practice preventive annual checkups of the condition of students' oral health. Each person has to understand the direct influence of bad hygiene of oral cavity and general health. Problems with teeth and the periodontium especially proved to be one of the reasons not only of bad state of health but also of the low level of the persons' self-confidence. Underestimating the importance of explanation to the population the fundamentals of general health care and oral health in particular, medical practitioners would not be successful in prophylactic work.

Diseases of the periodontium are widely spread in modern society. The number of gingivitis and periodontitis in young patients highly increased in recent decades. That is why, revelation and early treatment of periodontal diseases is important clinical as well as the ethic problem [5, p. 658-665]. Treatment of periodontal diseases in the initial and early stages prevents teeth loss in middle and older ages [3, p. 1-4; 6, p. 59-64].

Periodontal diseases are characterized by inflammatory and dystrophic-inflammatory processes in tooth surrounding tissues and the bone, which can be easily diagnosed in clinical observation. Important clinical problem, however, is diagnosing of persistent but not clinically pronounced inflammation in periodontal tissues. In many cases the situation is complicated by the absence of complaints from the patients, who are not aware of their problems and apply for the medical consultation only after noticing that their teeth start to be movable. That is why the task of each dentist noticing periodontal problems in his patient is to persuade him to start treatment immediately or consult the periodontal specialist. In the majority of clinical cases periodontal problems are connected with general health problems. A periodontologist is often the first medical specialist who can not only recommend but assure the patient to conduct examinations in other medical specialists. Sometimes not very complicated additional laboratory tests can be useful in the diagnosis of the periodontitis and other diseases (general blood test, X-ray examination, etc). Measurement of NO derivates in saliva, for example, can be greatly informative in diagnosing periodontal pathology in different stages of its development as well as in differentiation superficial inflammation of periodontal tissues in the case of gingivitis and profound destruction of gums and bone in the case of periodontitis. Nitric oxide (NO) is a free radical involved in the regulation of many physiological processes. Over recent years it has become apparent that NO has also important effects on bone cell function, specifically high levels of NO inhibit bone resorption and formation and may act to suppress bone turnover in severe inflammation [1, p. 255-261]. Inflammation is characterized by the raise of nitric anion concentration in biological fluids [2, p. 339-351]. Medical practitioner should be able to explain the necessity of each examination, being prescribe to the patient and discuss with the patient its results. Patient should feel himself not like a passive object in the treatment but take an active part in the process. Being acquainted with the treatment plan, presumable duration of the treatment and its' results, helps the patient to feel more comfortable and positive about the treatment procedures.

The aim of present investigation was the examination of patients (16-29 years old) and motivation them for treatment, when being at risk of periodontal and teeth diseases.

Matereials and methods. Under the supervision were 65 patients of 16-29 years old. All the patients were thoroughly motivated for the prophylactic dental examination and the following treatment in the case of necessity. Periodontal condition of all teeth has been conducted (including the estimation of pockets depth and presence of bleeding on probing) during the examination. Among the examined patients - 32 were diagnosed generalized periodontitis of the 1-st stage of heaviness (the depth of periodontal pockets up to3,5 mm), 5 patients - periodontitis of the 2-nd stage of heaviness (the depth of periodontal pockets 5-6 mm), 14 patients had chronic simple gingivitis, 14 persons - hypertrophic gingivitis. 10 healthy persons, 20-25 years old with intact periodontium formed the control group.

The level of inflammation in periodontal tissues has been estimated with bleeding index (Deneha, 1996) [4, p. 13-15]. Gingival bleeding varies in severity, duration and the ease with which it is provoked. The severity of the bleeding depends upon the intensity of the inflammation.

NO production was assessed indirectly by measuring accumulation of its stable metabolite - nitrite anion in 
saliva using Gries reaction. In our investigations NO production was studied in saliva, obtained in the quantity of one $\mathrm{ml}$ from each patient on an empty stomach.

Results. All the patients with periodontal diseases were given recommendations for the periodical clinical examination. Patients were persuaded to come for examinational check-ups. They have to be convinced by the doctor that the treatment of periodontal diseases in the initial and early stages prevents teeth loss in middle and older ages.

During the planned examinations all patients are distributed in three main groups.

Group one - healthy persons, without periodontal diseases - control visits once a year

Group two - patients with the high risk of periodontal diseases, local risk factors - maloocclusions and general factors - somatic pathology and patients treated for periodontal diseases after the achievement of the process stabilization for one year in gingivitis and two years in periodontitis.

Group three - patients treated for periodontal diseases. Subgroup A - patients with active processes being treated at present Subgroup B - patients which have finished the complex treatment of the disease achieving disease remission. The term remission means clinical improvement (absence of bleeding, periodontal pockets, normal gingival tissues but often with the gingival recession present after the treatment) and normalization of the bone structure. Patients in subgroup B have to come for examinational check-ups once a year in gingivitis, twice a year in periodontitis stage II and 3-4 times a year in periodontitis stage III. After the achieving of stable remission for one year in gingivitis and two years in periodontitis patients can be transformed from the group III B to the group II.

After the complex therapy in patients with generalized periodontitis has been carried out, the question, concerning the maintenance therapy with the account of the level of heaviness and character of the disease, arised. There is a great necessity to either eliminate the cause of the recurrence of disease or to minimize the influence of it. Two moments were to be precisely distinguished, thereat: the relapse of the inflammatory process in the periodontal tissues and the relapse of the periodontitis - the progressing of the periodontal, inflammatory-destructive process with the additional loss of the attachment and the formation (or deepening of the previously existing) periodontal pocket. As a rule, in the first clinical situation, it was quite enough to use the local measures (the tooth surface debridement, the polishing of the roots, the antiinflammatory therapy).

Treatment of all three groups has its peculiarities. There are different problems in group three A, were patients were undergoing active treatment procedures and need constant support, understanding and sympathy of the doctor and group three $\mathrm{B}$, in patients which have finished the complex treatment of the disease and must be more active themselves in following after- treatment regime and recommendations.

Once patients with periodontal disease have had their initial periodontal treatment, and if their periodontal disease has been halted, and they are able to maintain their teeth in a disease free state, they became more like a normal prophylaxis patient. However, since they have much more exposed root surface to scale, their "prophylaxis" is more complicated than a regular prophylaxis done on a patient without periodontal disease. These patients received a type of cleaning called a periodontal maintenance prophylaxis which is somewhat more labourintensive than a regular prophylaxis, and is generally done three or four times a year instead of twice a year as is done for patients without periodontal disease. Treatment for all stages of periodontal disease involves the thorough removal of all plaque and calculus that has adhered to the teeth. Even for patients who fit into the "prophylaxis patient" category above, calculus may build up very slightly below the gum line. This can be removed during a normal prophylaxis (scaling) without too much discomfort on the part of the patient. In patients were damage from gum disease is slight, this is done without local anesthesia. When the damage from periodontal disease is more pronounced, exposing more root structure above bone, the process of "root planing" is conducted. Root planing always requires local anesthesia. Painlessness of all dental procedures is a factor of the critical and decisive importance.

Since the ultrasonic and Vector methods are easily accepted by patients, in many cases it is possible to treat all of the subgingival and supragingival surfaces (often without using anaesthesia) in one session, which has a favorable effect on slowing the rate of reinfection. Ultrasonic instruments may be used for scaling, curetting, and removing stain. They work from physical vibrations of particles of matter, similar to sound waves, at frequencies ranging from 20000 to many million cycles per second (also referred to as Hertz $(\mathrm{Hz})$ ) above the range of human hearing. In periodontal instrumentation, ultrasonic units are composed of an electrical generator that delivers energy in the form of high-frequency (ultrasonic) vibrations to a handpiece into which a variety of specially designed tips may be inserted. The characteristic feature of the Vector system is a ring-shaped oscillating resonance body driven by an ultrasonic motor in a dental handpiece. Uncontrolled tumbling movements and mechanical vibrations from standard sonic and ultrasonic instruments and their self-oscillation characterized by oscillation loops and nodes as well as the resulting development of heat are therefore eliminated. High volumes of cooling water are not needed and the water supplied is not sprayed as an aerosol. The design of the Vector instruments is not subject to the usual restrictions (form and dimension, limited length or risk of breakage) and is exclusively oriented to the established clinical needs.

Additional, manual smoothing of the surfaces using hand instruments, such as curettes, as recommended for many traditional sonic or ultrasonic instruments, is not necessary for root surfaces treated with the Vector instrument, and should be avoided in order to prevent damage to the hard tooth tissue. If necessary, traditional polishing pastes and rotating brushes, etc. can also be used for removal of massive supragingival staining. The treatment is concluded beneficially by professional fluoride application to all the tooth surfaces, e.g., with highly concentrated fluoride gels.

All these details should be explained to the patients what make them much more positive motivated in the treatment. The patient has to be informed by the doctor 
about the treatment prognosis and be ready to consult his doctor in case of some unexpected problems. Therefore, good psychological contact between a doctor and a patient provides not only effective treatment of the periodontal disease but long-lasting stable situation after treatment which is not less important

The case of relapse of the periodontal disease demands the extra use of the surgical methods including the osteotrophic and stimulating therapy. The periodontal practice points out the five main causes leading to the renewal of the activity of the generalized periodontitis. They are as follows: the reduction of the non-specific resistance of the organism; the exacerbation of the somatic pathology with the aggravation of the secondary immunodeficiency that accompanies it; the long-term stress reactions; denaturalization of the nutrition as well as the poor oral hygiene. Therefore, taking into account the above-mentioned reasons, the use of the antibacterial, immunomodulatory, antioxidative or health-restorative therapy is strongly recommended along with the rotational diet and means for the deliverance of the internal medium acidosis.

The stress factor proved to be extremely significant in the cause of many human diseases including periodontal diseases. Periodontal treatment itself can be a stressful factor for the patient. In this situation the personality of the doctor, his (|her) attitude to the patient and the ability to support him in the long-term treatment of the periodontal disease are extremely valuable.

Conclusions. Annual prophylactic dental examinations in young patients are important task in the general medical health control system. They help to reveal not only diseases of teeth but also periodontal diseases. It is also an important ethical problem to persuade young persons in controlling the condition of oral health in annual preven- tive check-ups. Each person has to understand the direct influence of bad hygiene of oral cavity and general health. Problems with teeth and the periodontium especially proved to be one of the reasons not only of bad state of health but also of the low level of the persons' selfconfidence.

Medical practitioner should be able to explain the necessity of each examination, being prescribe to the patient and discuss with the patient its results. Patient should feel himself not like a passive object during the treatment but take an active part in the process. Periodontal treatment itself can be a stressful factor for the patient. In this situation the personality of the doctor, his (her) attitude to the patient and the ability to support him in the long-term treatment of the periodontal disease are extremely valuable.

Being acquainted with the treatment plan, presumable duration of the treatment and its' results, helps the patient to feel more comfortable and positive about the treatment procedures.

Patients have to be convinced by the doctor that the treatment of periodontal diseases in the initial and early stages prevents teeth loss in middle and older ages. The maintenance therapy with the account of the level of heaviness and character of the periodontal disease is utterly important and this fact should be explained to all patients.

The patient has to be informed by the doctor about the treatment prognosis and be ready to consult his doctor in case of some unexpected problems. Therefore, good psychological contact between a doctor and a patient provides not only effective treatment of the periodontal disease but long-lasting stable situation after treatment which is not less important.

\section{REFERENCES}

1. Nitric oxide and bone/Rob J. Van't Hof \& Stuart H. Ralston // Immunology, 2001.-№103.- P.- 255-261.

2. Arginin in medical practice/Yu.N. Stepanov, I.N. Kononov, A.I. Zhurbina, A.I. Filipova // Jornal medical sience of Ukraine, 2004.- №10 (2).- P. 339-351.

3. Teresa Bachanek, Barbara Hendzel, Ewa Wolańska, Volodymir Szybinsky, Roman Ogonovsky, Volodymir Hrynovets, et al. Prevalence of dental erosionamong 18-yearold ad olescentsin the borderland districts of Lviv (Ukraine) and Lublin (Poland) //Annals of Agricultural and Environmental Medicine. 2018. vol.25, №1.: 66-70.
4. Denega I.S. Informative value of bleeding index in periodontal diseases/I.S. Denega, S.J. Kukcta, V.S. Hrynovets B.C. Гриновець // Actual problems of dentistry, 1996.- №13.- P. 13-15.

5. L. Mitchell, D.A. Mitchell. Oksfordzki Podręcznik Stomatologii Klinicznej // Wydawnictwo Naukowe PWN.Warszawa, 1995.- 1075 p.

6. Ripetska O., Denega I., Hrynovets V., Hysyk M. Diseases of the periodontium (Etiology, pathogenesis, diagnosis, treatment.) - Lviv - Liga-Press - 2011. - 269 pp. 